Editorial

\title{
SARS COV 2 (COVID 19): Adapting to the Risk of Unknown Unknowns
}

\author{
Mahesh Goenka ${ }^{1}$ Nalini M. Guda ${ }^{2}$ \\ ${ }^{1}$ Department of Gastrosciences and Liver, Apollo Gleneagles \\ Hospitals Limited, Kolkata, West Bengal, India \\ ${ }^{2} \mathrm{GI}$ Associates, Milwaukee, Wisconsin, United States
}

J Digest Endosc 2020;11:1-2

What started as a small cluster of unknown cases of pneumonia in the Hubei province of China has rapidly evolved as a global pandemic affecting the entire world adversely both in health and economy. Scientists around the world have been acting swiftly from initial identification and genome sequencing to study the epidemiology, clinical features, disease containment, treatment, and eventual prevention/ eradication of the disease. While treatment options are promising, and vaccine appears a near distant reality, we as physicians are in the front line caring for the sick at a time when we also need to protect ourselves, the staff, and our families.

In this issue of the journal, we have compiled some very useful articles that pertain to gastroenterologists and especially endoscopists. The moot question is what are the implications of this disease to those working in the hospitals and centers where there is a risk of exposure and how to minimize risk? The effects of this current pandemic have wide ramifications affecting every facet of life. There are a lot of data pouring in as everyone is eager to share their experiences in the hope of achieving a collective victory over the battle Over the virus.

Key to any prevention or treatment would depend on the detailed knowledge of the virus. SARS-COV-2 has been identified, genomic sequencing has been done, and a lot of data have been collected. Epidemiology data including incubation time, infectivity, and implications of current methods of slowing down the contagion are critical as we race to find a cure or vaccine for eventual eradication. The series of articles by Sudipta Chowdhury and Usha Dutta and colleagues help us understand this process.

Though corona viruses including SARS-COV-2 predominantly cause respiratory symptoms, gastrointestinal illness has also been reported. The severity of gastrointestinal illness is variable and sometimes patients need endoscopic evaluation and/or interventions due to COVID or non-COVID-related illness. Understanding these issues and methods to ensure the safety of the endoscopist, staff, and the patient are critical. This will mean we critically assess the need for endoscopy and carefully weigh the risks and benefits and proceed with caution. Endoscopy units will have to ensure structural issues for adequate isolation, ventilation, and disinfection are in place. Because of risk of aerosolization during the procedures, the way we sedate and recover patients during this pandemic must be taken into serious consideration. When we are working with limitations in our personal protective equipment and other personnel and space constraints, we need to carefully allocate the resources for endoscopy. Choosing patients who need "time-sensitive procedures" is critical, and it helps to create a triage of algorithm so that we do not harm anyone by delaying care. It is important we recognize these issues and adjust our practices accordingly. Guidance on these issues based on what we know now is provided in this issue by Surinder Rana, Mathew Philip, Sundeep Lakhtakia, Deepak Gunjan, and their colleagues.

SARS-COV-2 does not just affect the luminal gastrointestinal system but can impact the liver as well. Most of the patients can have abnormalities in liver enzymes and the effects on the liver parenchyma appear to be immunemediated causing a hepatocellular pattern of liver injury. In this issue, Rakesh Kochhar, Anil Arora, and others discuss the pattern of gastrointestinal and liver injury including those at higher risk. We do have patients who are post liver transplant or those with non alcoholic fatty liver disease who would be at higher risk and these issues are detailed.

The current pandemic's negative impact unfortunately is not just limited to the health care system. Considering the significant infectivity and rapid spread of an infection which has a high morbidity and mortality, nations all over the world have implemented social distancing and other restrictive measures to "flatten the curve" and minimize the spread of the disease. India with a large population and limited resources has adopted the more stringent rules of isolation and social distancing. This has had a significant impact on the
Address for correspondence Mahesh Goenka, MD, DM, FACG, FASGE, AGAF, MRCP, Department of Gastrosciences and Liver, Apollo Gleneagles Hospitals Limited, Kolkata 700054, West Bengal, India (e-mail: mkgkolkata@gmail.com).
DOI https://doi.org/ 10.1055/s-0040-1712600 ISSN 0976-5042.
(C2020 Society of Gastrointestinal Endoscopy of India

\section{License terms}

$($ () (1) $\ominus \circledast$ 
economy and has made several smaller hospitals and endoscopic practices vulnerable and their viability is at risk. These and other financial aspects have been elaborated by Chirag Shah and Pankaj Dhawan.

SARS-COV-2 has been testing the limits of the health systems globally. Even nations that were thought to be better prepared have faced unsurmountable difficulties with supportive medical equipment and more importantly shortage of health care personnel. In this era of uncertainty, and limited treatment options, health care providers who are extending themselves thin also become legally vulnerable. There have been recent incidents where practitioners were injured by the patient's family. Legal issues surrounding the pandemic and how as a gastroenterologist one is covered and precautions one needs to take are highlighted here by Ashwini Sethya, Neeraj Nagpal, and team.

As a physician and a healer, we always look for the best treatment options. This is a novel virus and effective treatment options are limited. Various candidate drugs have been used without much or at best with limited evidence. In the normal times such use might be considered quackery at best, but current situations demand we explore every available option. Eradication of the disease will eventually mean vaccination or herd immunity. These issues are highlighted by Ashish Jha, Govind Makharia, and colleagues.

It is also time that we start looking beyond the present crisis. We hope we enter a next phase, where we would slowly but certainly move toward a less stressful situation. However, it is critical that this progression is scientific, cautiously optimistic with measured risks as we decide "a new normal" for the future. Randhir Sud and D.N. Reddy have addressed these vital aspects in this issue.

It is our sincere hope that we will overcome this pandemic collectively with shared knowledge, experience, and honest communications. As a physician and a leader of team, it is important you are well informed and make sound decisions protecting yourself, your families, and staff both physically and mentally while doing what we do the best-caring for our patients in the best way possible ensuring the most optimum outcomes.

We thank our authors, editorial team particularly Shivaraj Afzalpurkar, as well as our publishers for helping us to come out with this rapid communication. 